\author{
( С В.К. Федяева ${ }^{1,2}$, Е.А. Богова ${ }^{3}$, В.А. Петеркова ${ }^{3}$, О.Ю. Реброва ${ }^{3,4 *}$
}

'Центр экспертизы и контроля качества медицинской помощи, Москва, Россия

${ }^{2}$ Научно-исследовательский финансовый институт, Москва, Россия

${ }^{3}$ Национальный медицинский исследовательский центр эндокринологии, Москва, Россия

${ }^{4}$ Российский национальный исследовательский медицинский университет имени Н.И. Пирогова, Москва, Россия

Стремительный рост избыточной массы тела и ожирения у детей является глобальной проблемой здравоохранения. Наиболее эффективным считается проведение вмешательств по снижению распространенности ожирения до начала пубертатного возраста, однако результаты исследований не вполне согласованы. В данной работе выполнен метаанализ эффективности вмешательств с целью профилактики или коррекции избыточного веса и ожирения у детей препубертатного возраста. Из 31 Кокрейновского систематического обзора извлечены результаты 10 рандомизированных контролируемых испытаний и двух проспективных сравнительных исследований профилактических и лечебных немедикаментозных (коррекционных) вмешательств, применяемых для профилактики и лечения ожирения у детей 7-8 лет. Все исследования имели средний или высокий риск систематических ошибок. По результатам метаанализов обнаружено, что профилактика избыточного веса и ожирения путем рекомендаций модификации образа жизни эффективна (разница в снижении индекса массы тела -0,19, 95\% ДИ [-0,35; -0,03] по сравнению с отсутствием вмешательства) у детей 7-8 лет в случае их применения в течение 2-4 лет, но не в течение одного года и менее. Эффект немедикаментозных вмешательств у детей с избыточным весом или ожирением не выявлен.

КЛЮЧЕВЫЕ СЛОВА: дети; избыточная масса тела; ожирение; препубертатный возраст; метаанализ; профилактика; немедикаментозное лечение.

\title{
EFFICACY OF INTERVENTIONS FOR PREVENTION AND CORRECTION OF OVERWEIGHT AND OBESITY IN CHILDREN 7-8 YEARS OLD: A META-ANALYSIS
}

\author{
(c) Vlada K. Fediaeva ${ }^{1,2}$, Elena A. Bogova ${ }^{3}$, Valentina A. Peterkova ${ }^{3}$, Olga Yu. Rebrova ${ }^{3,4 *}$
}

${ }^{1}$ The Center for Healthcare Quality Assessment and Control, Moscow, Russia;

${ }^{2}$ Scientific and research financial institute, Moscow, Russia;

${ }^{3}$ Endocrinology Research Centre, Moscow, Russia;

${ }^{4}$ The Russian National Research Medical University named after N.I. Pirogov, Moscow, Russia

The rapid increase in overweight and obesity in children is a global public health problem. Interventions to reduce the prevalence of obesity before puberty are considered to be the most effective, but the results of trials are not consistent enough. We performed a meta-analysis of the efficacy of interventions to prevent or correct overweight and obesity in pre-pubertal children. Thirty-one Cochrane systematic reviews were analyzed, the results of 10 RCTs and two prospective comparative studies were extracted. All trials had an moderate or high risk of bias. According to the results of meta-analyzes, the prevention of overweight and obesity through recommendations of lifestyle modification is effective, the difference in a BMI decrease is - 0.19 , $95 \% \mathrm{Cl}[-0.35 ;-0.03]$, compared with the absence of intervention, in children $7-8$ years old if applied within 2-4 years, but not within one year or less. The effect of non-drug interventions in children with overweight or obesity was not revealed.

KEYWORDS: child; pre-pubertal; overweight; obesity; meta-analysis; prevention; treatment.

\section{ВВЕДЕНИЕ}

Стремительный рост избыточной массы тела и ожирения у детей является глобальной проблемой здравоохранения. Еще 40 лет назад ожирение диагностировалось не более чем у $1 \%$ всех детей в мире (5 млн девочек и 6 млн мальчиков), а в 2016 г. им страдали уже 5,6\% девочек (50 млн) и 7,8\% мальчиков (74 млн) [1]. Число детей и подростков с избыточной массой тела увеличилось на 213 млн в 2016 г. по сравнению с 1975 г. [1]. В России также отмечается рост распространенности детского ожире- ния. Если в 2005-2006 гг. ожирение диагностировалось у 1,2-4,8\%, а избыточная масса тела - у 4,6-11,8\% детей, то в 2014 гг. эти цифры увеличились в 2-3 раза, составив 5,5-6,06\% и 20,3-25,5\% соответственно, согласно данным национальных эпидемиологических исследований [2-6].

Возникновение ожирения в детском возрасте способствует не только его пролонгированию во взрослую жизнь, но и раннему развитию широкого спектра серьезных осложнений, таких как сахарный диабет, сердечно-сосудистые заболевания, нарушение дыхания во сне (апноэ), 
31 ссылка на систематические обзоры

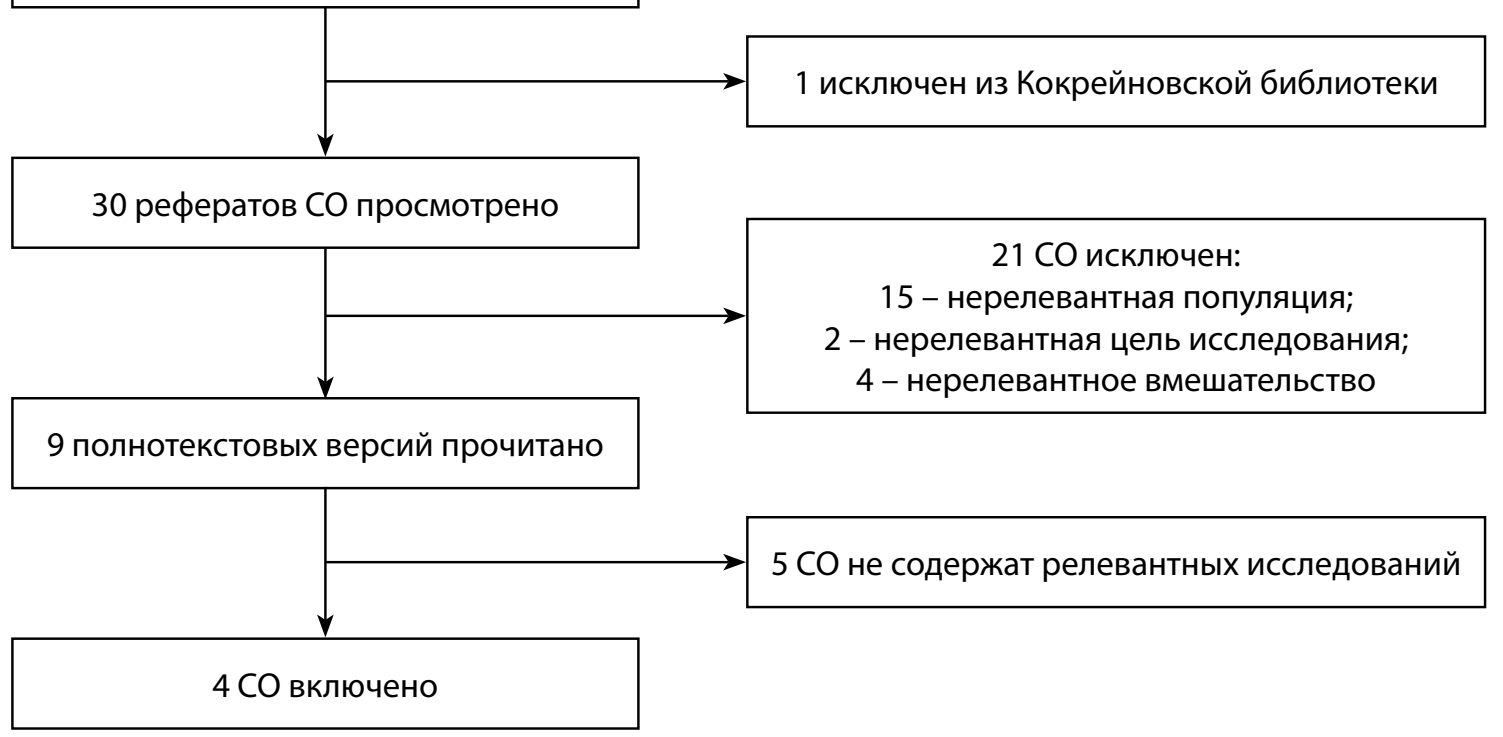

Рисунок 1. Потоковая диаграмма отбора систематических обзоров (СО) для дальнейшего рассмотрения.

патология пищеварительной системы и опорно-двигательного аппарата, психологические расстройства, что приводит к ухудшению качества жизни и преждевременной смертности [7]. Медикаментозная терапия ожирения у детей и подростков ограничена, а модификация образа жизни, включающая коррекцию рациона питания и повышение уровня физической активности, лежит в основе лечения и профилактики этого заболевания. Риск развития ожирения у детей увеличивается с возрастом, поэтому необходимо раннее принятие ответных мер на эпидемический процесс избыточного набора веса. Ввиду физиологических особенностей развития жировой массы, активный рост которой, начиная с 5-7 лет жизни, достигает максимума к периоду полового созревания, наиболее эффективным считается проведение вмешательств по снижению распространенности ожирения до начала пубертатного возраста [8-12], однако результаты исследований не вполне согласованы. В связи с этим целью данной работы являлось выполнение метаанализа эффективности вмешательств с целью профилактики или коррекции избыточного веса и ожирения у детей препубертатного возраста.

\section{МАТЕРИАЛЫ И МЕТОДЫ}

Выполнялся поиск систематических обзоров (СО) исследований профилактических и лечебных немедикаментозных (коррекционных) вмешательств, применяемых для профилактики и лечения ожирения у детей.

Источник информации: Кокрейновская библиотека систематических обзоров (cochranelibrary.com). Поисковый запрос: «children obesity» в полях Title, Abstract, Keyword. Поиск выполнен в июне 2019 г., повторен в сентябре 2019 г. По результатам поиска был найден 31 СО.

Далее выполнялся отбор СО с использованием следующих критериев исключения:

- отсутствие в СО исследований, изучающих популяцию детей 7-8 лет;

- отсутствие в СО исследований таких вмешательств, как поведенческая терапия, дополнительное педагогическое воздействие;
- медикаментозные или хирургические вмешательства для профилактики или лечения ожирения;

- отсутствие в СО проспективных сравнительных исследований.

На рис. 1 представлена потоковая диаграмма в соответствии с рекомендациями PRISMA, отражающая поиск и отбор СО.

После применения указанных критериев были отобраны 4 СО [13-16], при этом СО [16] являлся обновленной версией СО [13]: были включены дополнительные исследования, изучавшие вмешательства по профилактике ожирения у детей.

Поскольку ни в одном из найденных СО не были представлены отдельно сведения о результатах коррекции или профилактики избыточного веса и ожирения исключительно в популяции детей 7-8 лет (все результаты были представлены для смешанных возрастных групп), нами были просмотрены все включенные в отобранные СО оригинальные исследования. Всего в отобранные СО включено 55, 70, 18 и 153 исследования соответственно [13-16]. Из CO Waters et al., 2011 [13] были извлечены результаты 8 исследований [17-24], из CO Mead et al., 2018 [14] - информация об одном исследовании [25], из CO Martin et al., 2018 [15] - об одном исследовании [26], из CO Brown et al., 2019 [16] - о двух исследованиях $[27,28]$.

Краткая характеристика 12 отобранных исследований представлена в таблице 1. Десять исследований относились к профилактике ожирения, в них вмешательство применялось как к детям с нормальным весом, так и к детям с избыточным весом [17-24, $27,28]$. Восемь из этих 10 исследований имели дизайн рандомизированного контролируемого испытания (РКИ) или кластерного РКИ', два были сравнительными проспективными исследованиями. Два исследования

\footnotetext{
Кластерное РКИ - РКИ, в котором распределение по группам вмешательств проводится не на индивидуальном уровне, а на уровне групп пациентов. Пример: рандомизация школ, в результате которой в некоторых школах применяются исследуемые вмешательства, в других - вмешательства сравнения (в т.ч. отсутствие какого-либо вмешательства).
} 


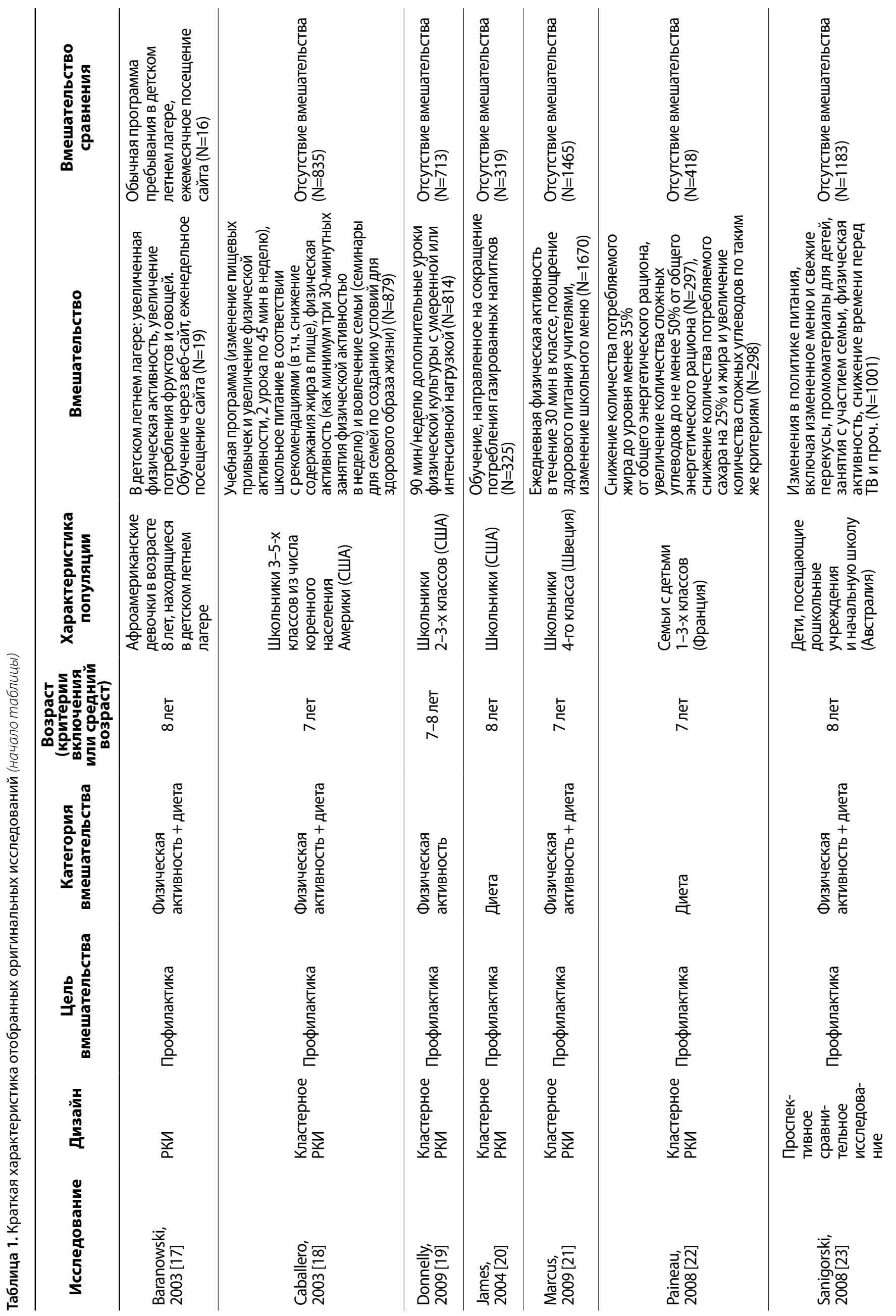




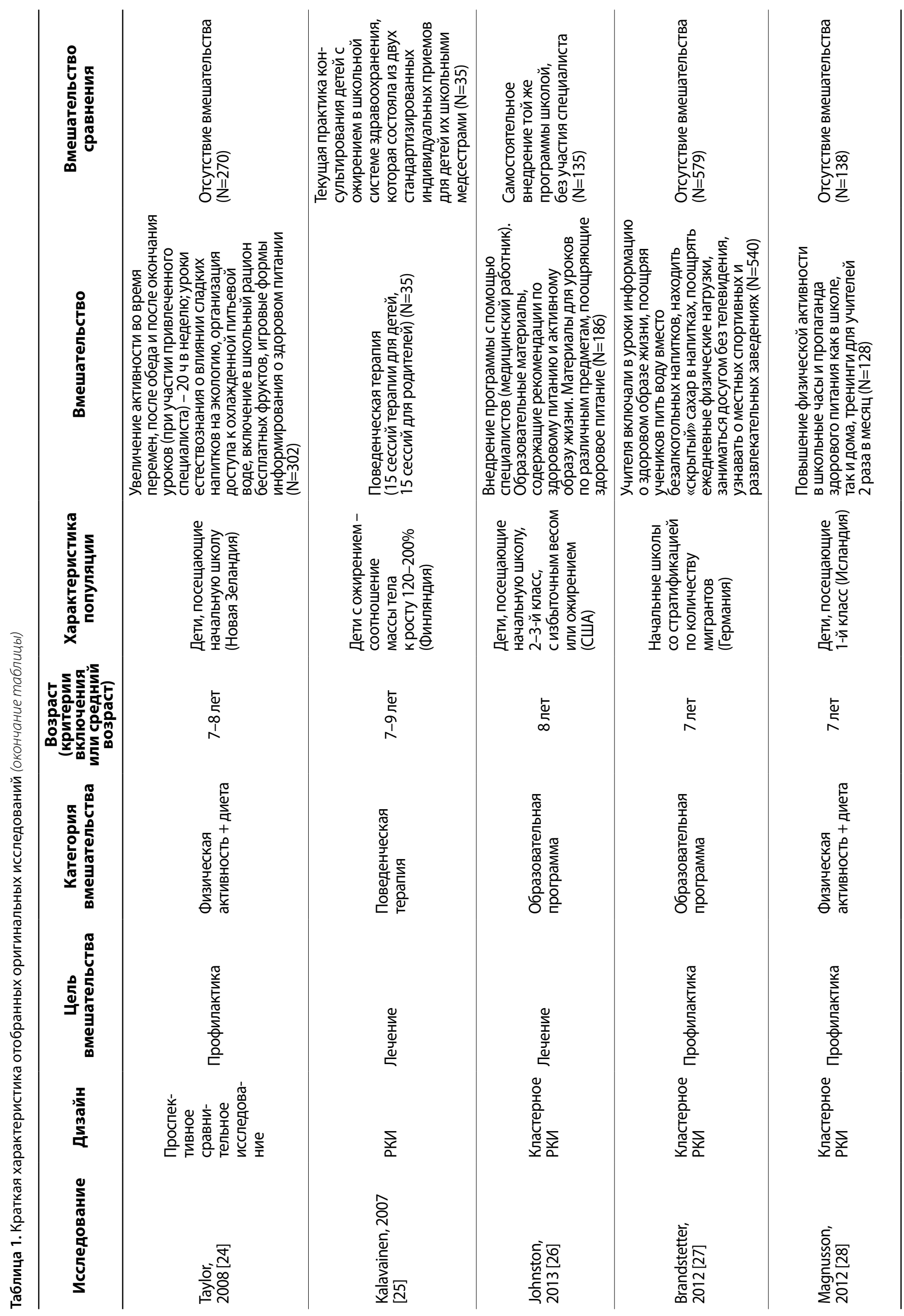


Таблица 2. Риски систематических смещений по доменам* [13-16] и итоговый риск**
Публикация

$\begin{array}{cc}\text { Генерация } & \text { Сокрытие } \\ \mathrm{P}^{* * * *} & \mathrm{P} \Pi^{* * *}\end{array}$
Маски-
рование
Выбывание
Полнота
Другие
Итоговый
риск

\begin{tabular}{ll|l|l|l|l|l|l}
\hline Baranowski, 2003 [17] & & & & & & & \\
Caballero, 2003 [18] & & & & & & & \\
Donnely, 2009 [19] & & & & & & & \\
James, 2004 [20] & & & & & & \\
Marcus, 2009 [21] & & & & & & \\
Paineau, 2008 [22] & & & & & & \\
Sanigorski, 2008 [23] & & & & & & \\
Taylor, 2008 [24] & & & & & & \\
Kalavainen, 2007 [25] & & & & & & \\
Johnston, 2013 [26] & & & & & & \\
Brandstetter, 2012 [27] & & & & & & & \\
Magnusson, 2012 [28] & & & & & & & \\
\hline
\end{tabular}

* З Зеленый цвет ячеек соответствует низкому, желтый - среднему, красный - высокому риску систематических ошибок по соответствующему домену.

** Итоговый риск систематических ошибок соответствует самому высокому риску в любом из доменов оценки.

*** РП - рандомизационная последовательность.

касались корригирующих вмешательств у детей с избыточной массой тела $[25,26]$, одно из них было РКИ, другое - кластерным РКИ.

Риски систематических смещений оценивались в соответствии с критериями Кокрейновского Сотрудничества [29, 30]. Для нерандомизированных исследований систематические смещения в первых двух доменах, связанных с рандомизацией, оценены по умолчанию как высокие. Оценка итогового риска систематических смещений также выполнена по правилу Кокрейновского Сотрудничества - он принят равным самому высокому риску из оценок по доменам $[29,30]$.

Метаанализ выполнялся в программе RevMan v.5.3 (The Cochrane Collaboration, 2014). Изменение индекса массы тела (ИМТ) ребенка (разница между исходным показателем и показателем по окончании периода наблюдения) за период вмешательства было основным критерием оценки эффективности вмешательств, соответственно метаанализ выполнялся по показателю разности средних изменений ИМТ в группах сравнения. Принято, что если разность средних между группами являлась отрицательным числом, исследуемое вмешательство имело больший эффект, чем контроль. Данные для анализа извлекались из СО, в ряде случаев - из оригинальных публикаций. До проведения метаанализа выполнялись оценка публикационного смещения (визуальная оценка по воронкообразным диаграммам рассеяния) и оценка гетерогенности эффектов (по критерию Хи-квадрат, также рассчитывался показатель гетерогенности $\mathrm{I}^{2}$ ). При отклонении гипотезы об однородности эффектов в метаанализе применялась модель случайных эффектов, в обратном случае модель фиксированного эффекта. Размеры эффектов взвешивались в соответствии с обратной величиной их дисперсии (inverse variance, IV).

\section{РЕЗУЛЬТАТЫ}

Результаты оценки авторами [13-16] риска систематических ошибок в доменах и наши оценки итогового риска представлены в табл. 2. Исследования профилактических вмешательств [17-24, 27, 28] имеют высокий или средний риск, оба исследования лечебных вмешательств $[25,26]$ - высокий риск систематических смещений.

Далее профилактические и лечебные немедикаментозные вмешательства анализировались нами отдельно. Также в связи с высокой вариабельностью длительности профилактических вмешательств мы разделили исследования на 2 группы: с длительностью менее 1 года (включительно) и длительностью 2-4 года.

На рис. 2 представлен метаанализ 3 исследований профилактических вмешательств длительностью 1 год и менее [17, 20, 22]. Публикационное смещение не выражено (рис. 2А). Эффекты в исследованиях разнонаправлены и высокогетерогенны, в связи с чем применялась модель случайных эффектов. Метаанализ не выявил эффект таких вмешательств (95\% ДИ для итоговой разности средних изменений ИМТ включает ноль).

В этот метаанализ невозможно было включить результаты еще одного РКИ с высоким риском систематических смещений Brandstetter 2012 [27], т.к. в публикации отсутствует информация об эффектах в группах и доступна лишь разность средних значений ИМТ и ее 95\% ДИ кокончанию периода наблюдения: $-0,06,95 \%$ ДИ $[-0,21 ; 0,10]$. Таким образом, результаты исследования [27] также демонстрируют отсутствие статистически значимых различий между группой вмешательства и группой сравнения в показателе изменения ИМТ к одному году наблюдения, т.е. являются согласованными с результатами выполненного нами метаанализа (95\% ДИ также включает ноль).

На рис. 3 представлен метаанализ 5 исследований профилактических вмешательств длительностью 
A

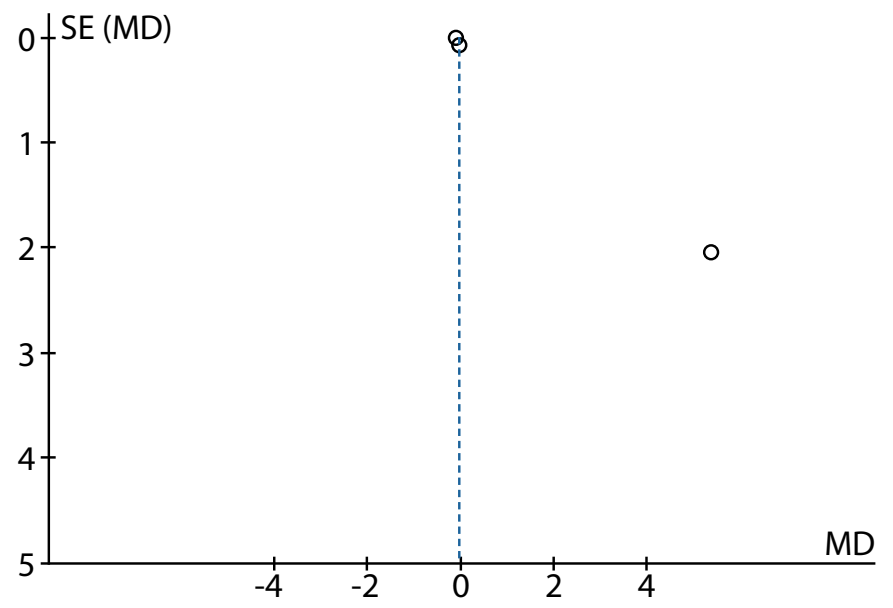

Б

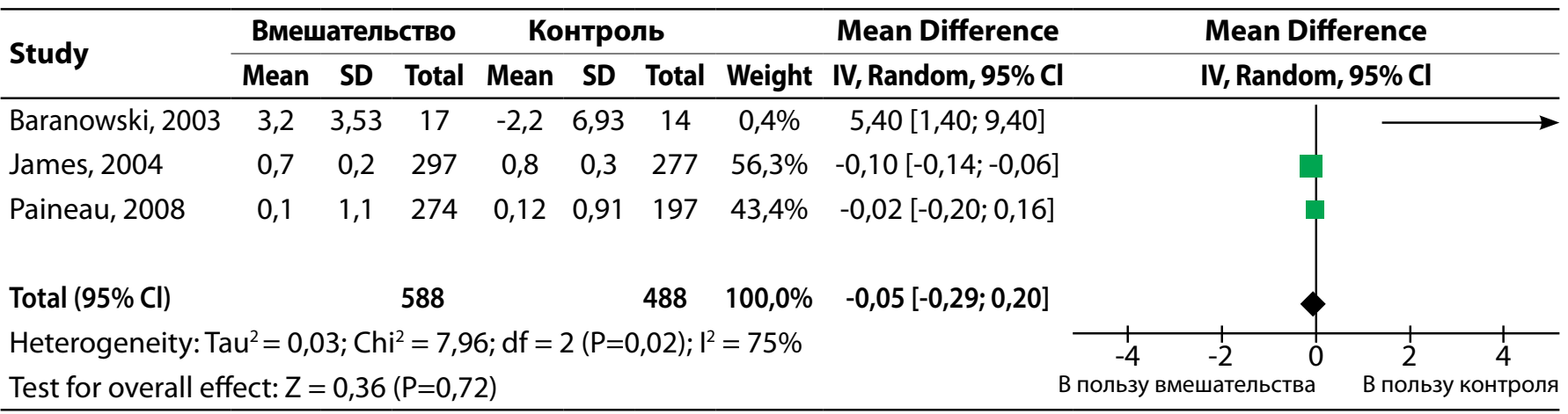

Рисунок 2. Метаанализ изменения индекса массы тела при применении профилактических вмешательств длительностью до 1 года. А - воронкообразный график для оценки публикационного смещения; Б - собственно метаанализ.

A

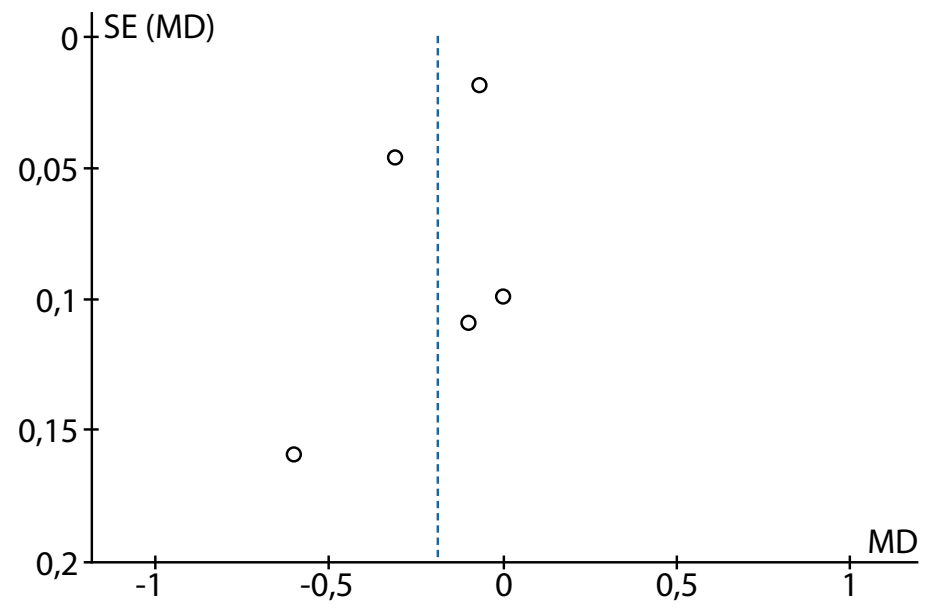

Б

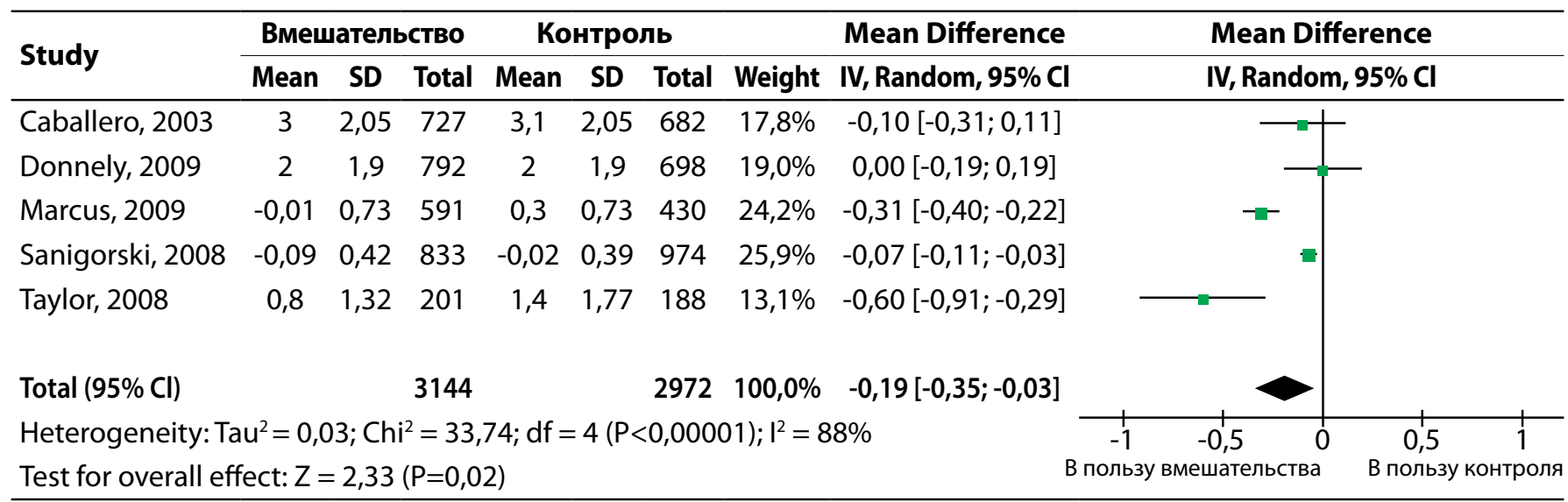

Рисунок 3. Метаанализ изменения индекса массы тела при применении профилактических вмешательств длительностью 2-4 года. А - воронкообразный график для оценки публикационного смещения; Б - собственно метаанализ. 
от 2 до 4 лет [18, 19, 21, 23, 24]. Публикационное смещение не выражено (рис. 3А). В этом случае эффекты однонаправленны, однако гетерогенность эффектов также высокая, поэтому вновь была использована модель случайных эффектов. В трех исследованиях был выявлен статистически значимый эффект вмешательства, а в двух исследованиях - нет, при этом метаанализ выявил наличие статистически значимого эффекта вмешательства величиной $-0,19,95 \%$ ДИ [-0,35; -0,03], хотя верхняя граница его 95\% ДИ и близка к нулю.

Результаты этого выполненного нами метаанализа не согласуются с результатами РКИ Magnusson 2012 с высоким риском систематических смещений [28], детальные результаты которого также не были приведены в СО и были извлечены нами из оригинальной публикации лишь в виде разности средних значений ИМТ и ее 95\% ДИ к окончанию периода наблюдения: 0,31, 95\% ДИ $[-0,16 ; 0,78]$. Таким образом, статистически значимые различия между группами к окончанию периода наблюдения 2 года в данном РКИ выявлены не были.

На рис. 4 представлен метаанализ 2 исследований лечебных немедикаментозных вмешательств, применявшихся к детям с ожирением [25, 26]. Публикационное смещение не выражено (рис. 4А). Следует отметить, что в одном исследовании отсутствовали статистически значимые различия между группой вмешательства и группой сравнения, а в другом исследовании (меньшем по числу наблюдений) такие различия были выявлены. При объединении результатов этих исследований в метаанализе эффект не выявлен (95\% ДИ включает ноль).

\section{ОБСУЖДЕНИЕ РЕЗУЛЬТАТОВ}

Для оценки роли профилактических и лечебных (немедикаментозных) мероприятий в снижении встречаемости избыточной массы и ожирения у детей (7-8 лет жизни) проанализированы Все $\mathrm{CO}(\mathrm{n}=31)$ Кокрейновской библиотеки, содержащие проспективные сравнительные исследования. Просмотрено 296 исследований, входящих в отобранные 4 СО, 12 из которых включены в метаанализ. Изучены не только результаты разнообразных коррекционных вмешательств, направленных как на изменение условий внешней среды (школы, дошкольные учреждения), так и на коррекцию образа жизни в семье, но и оценена их эффективность.

Известно, что по мере взросления детей повышается и заболеваемость ожирением, достигая пика дебюта к 7-8 годам жизни [7, 10-12, 31]. Также с возрастом снижается эффективность принимаемых мер по борьбе с ожирением [12]. Так, согласно данным Кокрейновского систематического обзора 2019 г., профилактические мероприятия по рационализации питания и физической активности приводят к более значимому снижению ИМТ у детей до 12 лет жизни, нежели в более старшем возрасте [16]. Поэтому для минимизации влияния метаболических, физиологических и психосоциальных различий для анализа нами была выбрана одна препубертатная возрастная группа детей (7-8 лет).

Эффекты от профилактических и лечебных воздействий оценивались раздельно. По результатам проведенного сравнительного анализа было выявлено, что лечение (немедикаментозное) ожирения у детей

A

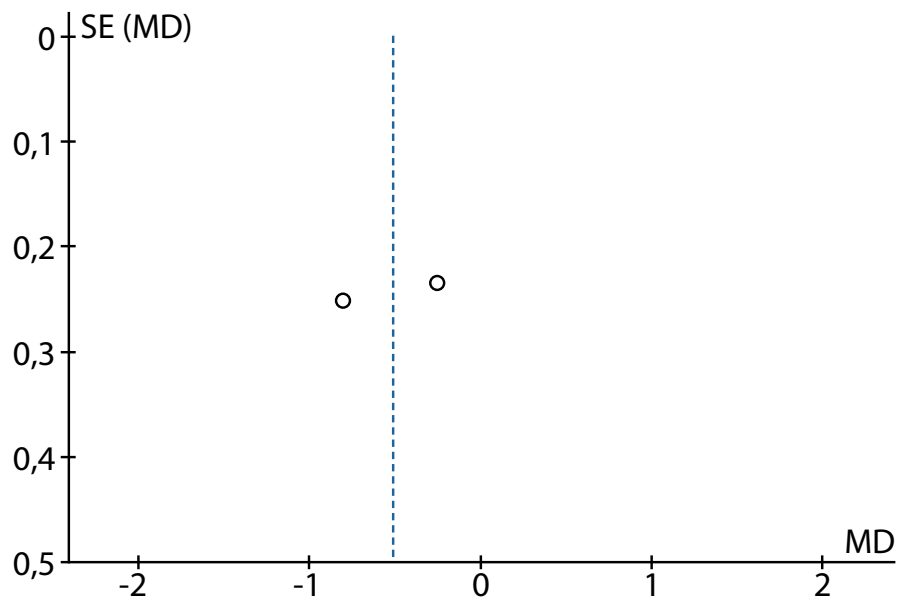

Б

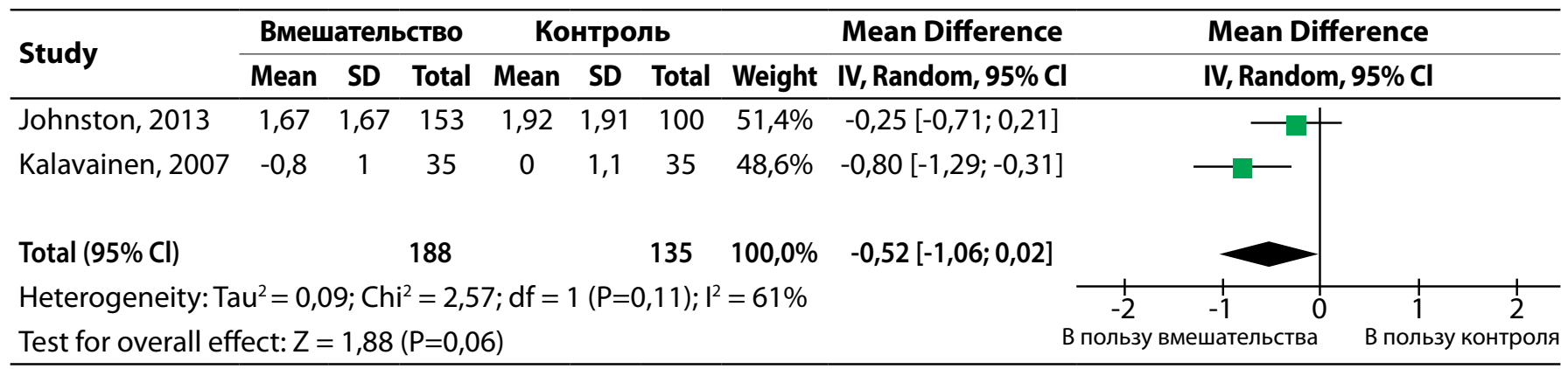

Рисунок 4. Метаанализ изменения индекса массы тела в период 0,5-2 года, лечебные вмешательства. А - воронкообразный график для оценки публикационного смещения; Б - собственно метаанализ. 
неэффективно, в отличие от профилактики, эффективность которой зависит от длительности воздействий. Эти результаты согласуются с данными исследований, показывающих слабый или умеренный краткосрочный эффект лечения ожирения у детей $[14,32]$. Также немедикаментозные вмешательства в анализируемых нами исследованиях отличались однонаправленностью воздействий $[25,26]$, носящих просветительский и образовательный характер.

Положительные результаты по снижению ИМТ по результатам нашего метаанализа были выявлены в исследованиях с превентивными вмешательствами продолжительностью более 2 лет и не отмечены при длительности профилактических мероприятий до 1 года. При изучении исследований, суммарно показавших свою эффективность $(n=5)$, было выявлено, что они были в основном направлены на изменение окружающей школьной среды. Так как большую часть времени дети проводят в образовательных учреждениях, школа может являться идеальной средой для борьбы с детским ожирением, оказывая влияние на рацион питания, характер физической активности, информированность о здоровом образе жизни детей и их семей. Действительно, многие школьные программы показывают свою эффективность в профилактике детского ожирения.

Однако только в 3 исследованиях [21, 23, 24] из 5 был выявлен статистически значимый эффект от принимаемых превентивных мер, что не повлияло тем не менее на общий положительный результат метаанализа. Эффективность вмешательств в этих работах может объясняться широкой разнонаправленностью оказываемых воздействий. Действительно, многие исследования свидетельствуют о преимуществах комплексных мер в отличие от мононаправленного воздействия на профилактику детского ожирения [19], что объясняется самим этиопатогенезом ожирения, являющимся мультифакторным заболеванием.

В работе Marcus (Швеция) в течение 4 лет уделялось внимание непосредственному изменению нескольких звеньев в школьной среде: физической активности (увеличение продолжительности ежедневной физической активности на 30 минут, сокращение экранного время до 30 минут в день), рациональному питанию (увеличение потребления разнообразных овощей, замена белого хлеба на цельнозерновой, сокращение содержания сахара в школьных обедах и полдниках за счет исключения из меню сладких напитков, соков, десертов, снижение содержания жира животного происхождения, в том числе в молочных продуктах), информированности и настороженности населения и работников школ по вопросам ожирения (дважды в год родители учащихся получали информационные бюллетени, проводились встречи с обученным школьным персоналом каждую четверть, школьные медсестры проходили обучение аспектам ожирения) [21]. Превентивная программа Sanigirski (Австралия) включала в себя не только комплексные школьные мероприятия, но и тесные взаимодействия с семьями учащихся [23]. В работе Taylor R.W. особое внимание уделялось внеклассной физической активности, наряду с изменением пищевого рациона [24].
Важности физической активности в вопросах профилактики ожирения у детей уделяется особое внимание. Так, согласно результатам Кокрейновского метаанализа 2019 г., именно физическая активность позволила снизить риск развития ожирения в возрастной группе детей от 6 до 12 лет [11]. Эти результаты подтверждаются и данными других работ, показывающих ведущую роль физической активности в борьбе с ожирением у детей $[33,34]$. В исследованиях с отсутствием статистически значимого эффекта вмешательств $[18,19]$ оптимизация физической нагрузки также была включена в превентивную программу, но в работе Caballero она являлась единственной стратегией (продолжительность не более 150 мин в неделю), а в исследовании Donelly [19] характеризовалась небольшой длительностью (не более 90 мин в неделю) и отсутствием поддержания внеклассной физической активности (занятия физкультурой были включены только в школьные уроки). В исследовании Marcus C. [21], показавшем свою эффективность в снижении детского ожирения, ежедневная продолжительность физических нагрузок в школе была увеличена дополнительно на 30 минут, а длительность компьютерных игр ограничена до 30 минут в день. В работах с положительным эффектом от вмешательств Taylor [24] и Sanigorski [23], помимо занятий физкультурой в школе, поощрялась и была увеличена внеклассная двигательная активность, в том числе в игровой форме. Компонент удовольствия в физической активности может играть важную роль в успешности мер в профилактике ожирения, что хорошо показано в работе Liu Z. и созвучно с результатами других исследований, показывающих, что сниженная мотивация и удовольствие от физической активности являются барьером для занятий физкультурой [34].

У настоящего метаанализа существует ряд методологических ограничений, вследствие которых его результаты необходимо интерпретировать с осторожностью:

- использование вторичных источников информации для поиска оригинальных публикаций;

- отсутствие исследований высокого методологического качества;

- несогласованность результатов отдельных исследований, наличие статистической гетерогенности; использование средних при невозможности убедиться в нормальности распределения данных (в оригинальных публикациях данные о том, что признаки распределены нормально, не представлены);

- включение в один анализ исследований с различными вмешательствами и периодами наблюдения.

\section{ЗАКЛЮЧЕНИЕ}

Профилактика избыточного веса и ожирения путем рекомендаций модификации образа жизни эффективна (разница в снижении ИМТ -0,19, 95\% ДИ [-0,35; -0,03] по сравнению с отсутствием вмешательства) у детей 7-8 лет в случае их применения в течение 2-4 лет, но не в течение одного года и менее, однако эти результаты получены в исследованиях с высоким и средним риском систематических смещений. Эффект немедикаментозных вмешательств у детей с избыточным весом или ожирением не выявлен. Несмотря на ограничения 
нашего исследования, можно заключить, что применение комплексных превентивных мер длительностью не менее 2 лет у детей препубертатного возраста (7-8 лет) показывает свою эффективность в снижении распространенности детского ожирения, в отличие от лечебных мероприятий. Эта информация может быть полезна для научных, образовательных, государственных организаций, семей, гражданского общества. Проблема детского ожирения с каждым годом все больше приобретает характер эпидемии, остановить которую можно только начав с ранней профилактики. Сложность проблемы ожирения требует комплексного подхода с привлечением всех сфер общества.

\section{ДОПОЛНИТЕЛЬНАЯ ИНФОРМАЦИЯ}

Источник финансирования. Подготовка и публикация рукописи проведены на личные средства авторского коллектива.

Конфликт интересов. Авторы декларируют отсутствие явных и потенциальных конфликтов интересов, связанных с публикацией настоящей статьи.

Участие авторов. В.К. Федяева - получение и анализ данных, интерпретация результатов, написание статьи; Е.А. Богова - интерпретация результатов, редактирование статьи; В.А. Петеркова - разработка концепции исследования, редактирование статьи; О.Ю. Реброва - разработка дизайна исследования, редактирование статьи. Все авторы прочли и одобрили финальную версию статьи.

\section{СПИСОК ЛИТЕРАТУРЫ | REFERENCES}

1. Abarca-Gómez L, Abdeen ZA, Hamid ZA, et al. Worldwide trends in body-mass index, underweight, overweight, and obesity from 1975 to 2016: a pooled analysis of 2416 populationbased measurement studies in 128.9 million children, adolescents, and adults. Lancet. 2017;390(10113):2627-2642 doi: https://doi.org/10.1016/s0140-6736(17)32129-3

2. World Health Organization Europe. The challenge of obesity in the WHO European Region. Fact Sheet EURO/13/05. Copenhagen, Bucharest, 2005 September 12.

3. Дедов И.И., Мельниченко Г.А., Бутрова С.А., и др. Ожирение у подростков в России // Ожирение и метаболизм. - 2006. - Т. 3. №4. - C. 30-34. [Dedov II, Mel'nichenko GA, Butrova SA, et al. Ozhirenie u podrostkov v Rossii. Obesity and metabolism. 2006;3(4):30-34. (In Russ.)] doi: https://doi.org/10.14341/2071-8713-5141

4. Тутельян В.А., Батурин А.К., Конь И.Я., и др. Распространенность ожирения и избыточной массы тела среди детского населения РФ: мультицентровое исследование // Педиатрия. Журнал им. Г.Н. Сперанского. — 2014. - Т. 93. - №5. - С. 28-31. [Tutel'yan VA, Baturin AK, Kon' IY, et al. Rasprostranennost' ozhireniya i izbytochnoy massy tela sredi detskogo naseleniya RF: mul'titsentrovoe issledovanie. Pediatriia. 2014;93(5):28-31. (In Russ.)]

5. Ахмедова Р.М., Софронова Л.В., Трефилов Р.Н. Распространенность и гендерные особенности ожирения у подростков Перми // Вопросы современной педиатрии. - 2014. - Т. 13. №5. - C. 37-41. [Ahmedova RM, Sofronova LV, Trefilov RN. Prevalence and gender characteristics of obesity in adolescents in the city of Perm. Current pediatrics. 2014;13(5):37-41. (In Russ.)] doi: https://doi.org/10.15690/vsp.v13i5.1148

6. Соболева Н.П., Руднев С.Г., Николаев Д.В., и др. Биоимпедансный скрининг населения России в центрах здоровья: распространенность избыточной массы тела и ожирения // PMЖ. — 2014. - T. 20. — №4. - C. 4-13. [Soboleva NP, Rudnev SG, Nikolayev DV, et al. The bio-impedance screening of population in health centers: prevalence of surplus body mass and obesity. RMZh. 2014;20(4):4-13. (In Russ.)]

7. Павловская Е.В. Коморбидная патология при ожирении у детей: клинико-метаболическая характеристика, оценка эффективности диетотерапии: Дис. ... д-ра мед. наук. - М.; 2020. [Pavlovskaya EV. Komorbidnaya patologiya pri ozhirenii u detey: klinikometabolicheskaya kharakteristika, otsenka effektivnosti dietoterapii. [dissertation] Moscow; 2020. (In Russ.)]

8. MacLean PS, Higgins JA, Giles ED, et al. The role for adipose tissue in weight regain after weight loss. Obes Rev. 2015;16 Suppl 1:45-54 doi: https://doi.org/10.1111/obr.12255

9. Стародубова А.В. Факторы риска, особенности формирования и течения сердечно-сосудистых заболеваний у женщин с ожирением разных возрастных групп и возможности их коррекции: Дис. ... д-ра мед. наук. - М.; 2015. [Starodubova AV. Faktory riska, osobennosti formirovaniya i techeniya serdechnososudistykh zabolevaniy u zhenshchin s ozhireniem raznykh vozrastnykh grupp i vozmozhnosti ikh korrektsii. [dissertation] Moscow; 2015. (In Russ.)]

10. Камалова А.А. Современные подходы к профилактике ожирения у детей // Российский вестник перинатологии и педиатрии. — 2016. — Т. 61. — №6. — C. 43-48. [Kamalova AA.
Current approaches to preventing childhood obesity. Rossiiskii vestnik perinatologii i pediatrii. 2016;61(6):43-48. (In Russ.)] doi: https://doi.org/10.21508/1027-4065-2016-61-6-43-48

11. Weihrauch-Bluher S, Schwarz P, Klusmann JH. Childhood obesity: increased risk for cardiometabolic disease and cancer in adulthood. Metabolism. 2019;92:147-152. doi: https://doi.org/10.1016/j.metabol.2018.12.001

12. Kim J, Must A, Fitzmaurice GM, et al. Incidence and remission rates of overweight among children aged 5 to 13 years in a district-wide school surveillance system. Am J Public Health. 2005;95(9):1588-1594. doi: https://doi.org/10.2105/AJPH.2004.054015

13. Waters E, de Silva-Sanigorski A, Hall BJ, et al. Interventions for preventing obesity in children. Cochrane Database Syst Rev. 2011(12):CD001871. doi: https://doi.org/10.1002/14651858.CD001871.pub3

14. Mead E, Brown T, Rees K, et al. Diet, physical activity and behavioural interventions for the treatment of overweight or obese children from the age of 6 to 11 years. Cochrane Database Syst Rev. 2017;6:CD012651. doi: https://doi.org/10.1002/14651858.CD012651

15. Martin A, Booth JN, Laird Y, et al. Physical activity, diet and other behavioural interventions for improving cognition and school achievement in children and adolescents with obesity or overweight. Cochrane Database Syst Rev. 2018;3:CD009728. doi: https://doi.org/10.1002/14651858.CD009728.pub4

16. Brown T, Moore TH, Hooper L, et al. Interventions for preventing obesity in children. Cochrane Database Syst Rev. 2019;7:CD001871. doi: https://doi.org/10.1002/14651858.CD001871.pub4

17. Baranowski T, Baranowski JC, Cullen KW, et al. The fun, food, and fitness project (FFFP): the Baylor GEMS pilot study. Ethn Dis. 2003;13(1 Suppl 1):S30-39

18. Caballero B, Clay T, Davis SM, et al. Pathways: a school-based, randomized controlled trial for the prevention of obesity in American Indian schoolchildren. Am J Clin Nutr. 2003;78(5):1030-1038. doi: https://doi.org/10.1093/ajcn/78.5.1030

19. Donnelly JE, Greene JL, Gibson CA, et al. Physical Activity Across the Curriculum (PAAC): a randomized controlled trial to promote physical activity and diminish overweight and obesity in elementary school children. Prev Med. 2009:49(4):336-341. doi: https://doi.org/10.1016/j.ypmed.2009.07.022

20. James J, Thomas P, Cavan D, Kerr D. Preventing childhood obesity by reducing consumption of carbonated drinks: cluster randomised controlled trial. BMJ. 2004:328(7450):1237. doi: https://doi.org/10.1136/bmj.38077.458438.EE

21. Marcus C, Nyberg G, Nordenfelt A, et al. A 4-year, cluster-randomized, controlled childhood obesity prevention study: STOPP. Int J Obes (Lond). 2009;33(4):408-417. doi: https://doi.org/10.1038/ijo.2009.38

22. Paineau DL, Beaufils F, Boulier $A$, et al. Family dietary coaching to improve nutritional intakes and body weight control: a randomized controlled trial. Arch Pediatr Adolesc Med. 2008;162(1):34-43. doi: https://doi.org/10.1001/archpediatrics.2007.2

23. Sanigorski AM, Bell AC, Kremer PJ, et al. Reducing unhealthy weight gain in children through community capacitybuilding: results of a quasi-experimental intervention program, Be Active Eat Well. Int J Obes (Lond). 2008;32(7):1060-1067. doi: https://doi.org/10.1038/ijo.2008.79 
24. Taylor RW, McAuley KA, Barbezat W, et al. Two-year follow-up of an obesity prevention initiative in children: the APPLE project. Am J Clin Nutr. 2008;88(5):1371-1377. doi: https://doi.org/10.3945/ajcn.2007.25749

25. Kalavainen MP, Korppi MO, Nuutinen OM. Clinical efficacy of groupbased treatment for childhood obesity compared with routinely given individual counseling. Int J Obes (Lond). 2007;31(10):1500-1508. doi: https://doi.org/10.1038/sj.ijo.0803628

26. Johnston CA, Moreno JP, El-Mubasher A, et al. Impact of a schoolbased pediatric obesity prevention program facilitated by health professionals. J Sch Health. 2013;83(3):171-181. doi: https://doi.org/10.1111/josh.12013

27. Brandstetter S, Klenk J, Berg S, et al. Overweight prevention implemented by primary school teachers: a randomised controlled trial. Obes Facts. 2012;5(1):1-11. doi: https://doi.org/10.1159/000336255

28. Magnusson KT, Hrafnkelsson H, Sigurgeirsson I, et al. Limited effects of a 2-year school-based physical activity intervention on body composition and cardiorespiratory fitness in 7-year-old children. Health Educ Res. 2012;27(3):484-494. doi: https://doi.org/10.1093/her/cys049

29. Реброва О.Ю., Федяева В.К., Хачатрян Г.Р. Адаптация и валидизация вопросника для оценки риска систематических ошибок в рандомизированных контролируемых испытаниях // Медицинские технологии. Оценка и выбор. - 2015. T. 19. - №1. - C. 9-17. [Rebrova OY, Fedyaeva VK, Khachatryan GR. Adaptation and Validation of the Cochrane Questionnarie to Assess Risks of Bias in Randomized Controlled Trials. Medical technologies. 2015;19(1):9-17. (In Russ.)]
30. Training cochrane.org [Internet]. Julian PT Higgins, Jelena Savović, Matthew J Page, et al. Chapter 8: Assessing risk of bias in a randomized trial [cited 2020 Aug 10]. Available from: https://training.cochrane.org/handbook/current/chapter-08.

31. Витебская А.В., Писарева Е.А., Попович А.В. Образ жизни детей и подростков с ожирением. Результаты анкетирования пациентов и их родителей // Ожирение и метаболизм. - 2016. — Т. 13. №2. - C. 33-40. [Vitebskaya AV, Pisareva EA, Popovich AV. Lifestyle in children and adolescents with obesity: results of the survey of patients and their parents. Obesity and metabolism. 2015;13(2):33-40. (In Russ.)] doi: https://doi.org/10.14341/omet2016233-40

32. McGovern L, Johnson JN, Paulo R, et al. Treatment of Pediatric Obesity: A Systematic Review and Meta-Analysis of Randomized Trials. J Clin Endocrinol Metab. 2008;93(12):4600-4605. doi: https://doi.org/10.1210/jc.2006-2409

33. Ануфриева Е.В. Основные закономерности формирования, прогнозирования здоровья детей школьного возраста и совершенствование системы медицинского обеспечения в образовательных организациях: Дис. ... д-ра мед. наук. Екатеринбург; 2019. [Anufrieva EV. Osnovnye zakonomernosti formirovaniya, prognozirovaniya zdorov'ya detey shkol'nogo vozrasta i sovershenstvovanie sistemy meditsinskogo obespecheniya v obrazovatel'nykh organizatsiyakh. [dissertation] Ekaterinburg; 2019. (In Russ.)]

34. Liu Z, Xu HM, Wen LM, et al. A systematic review and metaanalysis of the overall effects of school-based obesity prevention interventions and effect differences by intervention components. Int J Behav Nutr Phys Act. 2019;16(1):95 doi: https://doi.org/10.1186/s12966-019-0848-8

\section{ИНФОРМАЦИЯ ОБ АВТОРАХ [AUTHORS INFO]:}

*Реброва Ольга Юрьевна, д.м.н. [Olga Yu. Rebrova, PhD]; адрес: Россия, 117036, Москва, ул. Дм. Ульянова, д. 11 [address: 11 Dm. Ulyanova street, 117036 Moscow, Russia]; ORCID: https://orcid.org/0000-0002-6733-0958; eLibrary SPIN: 7360-3254; e-mail: o.yu.rebrova@gmail.com

Федяева Влада Константиновна [Vlada K. Fediaeva]; ORCID: https://orcid.org/0000-0002-7730-1237; eLibrary SPIN: 7202-4371; e-mail: vlada.fedyaeva@gmail.com

Богова Елена Ахсарбековна, к.м.н. [Elena A. Bogova, MD, PhD]; ORCID: https://orcid.org/0000-0001-7445-4871; eLibrary SPIN: 2273-4237; e-mail: eabogova@gmail.com

Петеркова Валентина Александровна, А.М.н., профессор [Valentina A. Peterkova, MD, PhD, Professor]; ORCID: https://orcid.org/0000-0002-5507-4627; eLibrary SPIN: 4009-2463; email: peterkovava@hotmail.com

*Автор, ответственный за переписку / Corresponding author.

\section{ЦИТИРОВАТЬ:}

Федяева В.К., Богова Е.А., Петеркова В.А., Реброва О.Ю. Метаанализ эффективности вмешательств для профилактики и коррекции избыточного веса и ожирения у детей 7-8 лет // Ожирение и метаболизм. - 2020. - Т. 17. - №2. - С. 115-124. doi: https://doi.org/10.14341/omet12120

\section{TO CITE THIS ARTICLE:}

Fediaeva VK, Bogova EA, Peterkova VA, Rebrova OY. Efficacy of interventions for prevention and correction of overweight and obesity in children 7-8 years old: a meta-analysis. Obesity and metabolism. 2020;17(2):115-124. doi: https://doi.org/10.14341/omet12120 